\title{
Application of Digital Anti-Coincidence Counting Method for Primary Activity Determination of ${ }^{59} \mathrm{Fe}$
}

\author{
A. Agusbudiman ${ }^{1,3 \star}$, K.B. Lee ${ }^{2,3}$ and J.M. Lee ${ }^{2,3}$ \\ ${ }^{I}$ Center for Technology of Radiation Safety and Metrology, National Nuclear Energy Agency, \\ Jl. Lebak Bulus Raya No. 49, Jakarta 12440, Indonesia \\ ${ }^{2}$ Korea Research Institute of Standards and Science, 267 Gajeong-ro, Yuseong-gu, Daejeon 34113, Republic of Korea \\ ${ }^{3}$ Korea University of Science and Technology, 217 Gajeong-ro, Yuseong-gu, Daejeon 34113, Republic of Korea
}

\section{ARTICLE INFO}

\section{Article history:}

Received 15 January 2018

Received in revised form 26 February 2019

Accepted 4 March 2019

\section{Keywords:}

${ }^{59}$ Fe standardization

Anti- coincidence counting

Liquid scintillation

Digital coincidence counting

Efficiency extrapolation method

\begin{abstract}
A B S T R A C T
As a radiopharmaceutical, the use of ${ }^{59} \mathrm{Fe}$ is classified as a high pharmaceutical risk product. Therefore, a standard reference for activity measurement of ${ }^{59} \mathrm{Fe}$ is necessary to ensure its metrological aspect. This paper describes an alternative method for primary activity determination of ${ }^{59} \mathrm{Fe}$ for establishing a standard reference. The ${ }^{59} \mathrm{Fe}$ solution was prepared using two different cocktails and measured by the $4 \pi \beta(\mathrm{LS})-\gamma$ counting system using a digital anti-coincidence counting method with emulated live-time of the extending dead-time. A final activity results at the reference time for the two samples series are $(473.32 \pm 2.55)$ $\mathrm{kBq} / \mathrm{g}$ and $(477.14 \pm 2.42) \mathrm{kBq} / \mathrm{g}$ with quoted uncertainty evaluated at $k=1$. The final activity was compared to the value obtained from the other two coincidence counting method and found to be in a good agreement within its uncertainty value.
\end{abstract}

(C) 2019 Atom Indonesia. All rights reserved

\section{INTRODUCTION}

Ferrous-59 $\left({ }^{59} \mathrm{Fe}\right)$ is widely used in the nuclear medicine field as a radiopharmaceutical tracer for studying iron metabolism. As a radiopharmaceutical, ${ }^{59} \mathrm{Fe}$ is classified as high pharmaceutical risk product. Therefore, it must be very carefully characterized in terms of three main parameters: physicochemical, metrological, and biological [1]. The metrological parameter, in particular, precise measurements of activity, is crucial as it directly determines the effective dose given to the patient.

Radionuclide ${ }^{59} \mathrm{Fe}$ decays with a half-life of 44.494(12) days, by several beta minus emissions to the ground state and to four excited states of ${ }^{59} \mathrm{Co}$, mainly to the 1099 and $1291 \mathrm{keV}$ [2]. A simplified decay scheme of ${ }^{59} \mathrm{Fe}$ is shown in Fig. 1.

The nature of ${ }^{59} \mathrm{Fe}$ makes it possible to conduct an activity standardization with several methods. Most of the primary activity standardization for ${ }^{59} \mathrm{Fe}$ was conducted using the

* Corresponding author.

E-mail address: agusbudiman @ batan.go.id

DOI: https://doi.org/10.17146/aij.2019.812
$4 \pi \beta-\gamma$ coincidence counting method, which has been confirmed to give a good result as described in [3-7].

Another method reported by Kossert and Nähle (2014), after a successful activity standardization of ${ }^{59} \mathrm{Fe}$, is based on liquid scintillation (LS) counters using CIEMAT/ NIST and TDCR method [8]. In this work, we applied a digital anti-coincidence counting method with emulated live-time of the extending dead-time to determine the absolute activity of ${ }^{59} \mathrm{Fe}$.

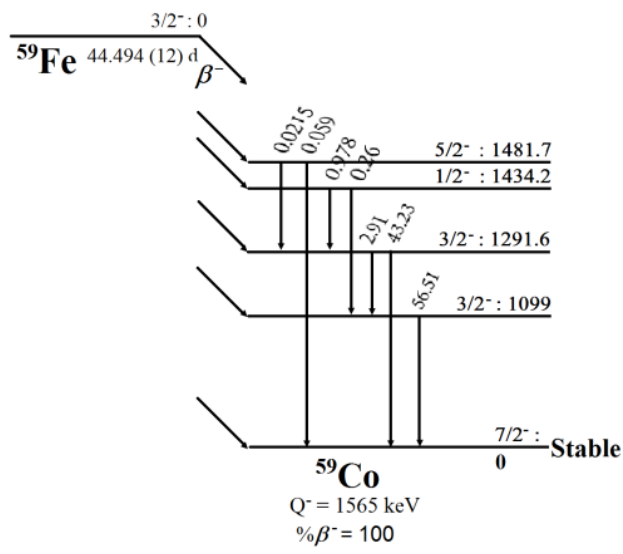

Fig. 1. Simplified decay scheme of ${ }^{59} \mathrm{Fe}$. 
The anti-coincidence method has been successfully applied to determine the activity of other radionuclides, such as ${ }^{129} \mathrm{I},{ }^{131} \mathrm{I},{ }^{18} \mathrm{~F},{ }^{68} \mathrm{Ga}$, and ${ }^{134} \mathrm{Cs}$ [9-13]. These results were also compared to other methods including the coincidence counting method, and they were found to give equivalent results.

\section{THEORY}

The anti-coincidence counting method is a complement of the coincidence counting method with no basic difference between the two methods. Therefore, the count rates of beta-ray $\left(\rho_{\beta}\right)$, gammaray $\left(\rho_{\gamma}\right)$, and beta-gamma coincidence $\left(\rho_{\beta \gamma}\right)$ are still necessary to be determined. Unlike the coincidence counting method, in anti-coincidence the $\rho_{\beta \gamma}$ is obtained from the difference between the total gamma-ray count rate $\rho_{\gamma}$ and the uncorrelated non-coincident gamma-ray count rate, $\rho_{a}$, as described in Eq. (1).

$$
P_{\beta \gamma}=\rho_{\gamma}-\rho_{\alpha}
$$

Taking Eq. (1) into the basic coincidence equation, the source activity value is therefore given by:

$$
A=\frac{\rho_{\beta} \rho_{\gamma}}{\rho_{\gamma}-\rho_{\alpha}}
$$

A linear extrapolation of $\rho_{\beta} \rho_{\gamma} / \rho_{\beta \gamma}$ as a function of $\rho_{\gamma} / \rho_{\beta \gamma}-1$ will yield the source activity $A$.

One complicated part of the application of anti-coincidence counting is the correction for deadtime losses as the dead-time loss in beta channel and coincidence channel do not compensate to some extent as in coincidence counting. The live-timed anti-coincidence counting with extendable deadtime circuitry is believed to be able to overcome the problem with dead-time losses.

\section{EXPERIMENTAL METHODS}

The master solution of ${ }^{59} \mathrm{Fe}$ in the chemical form of $\mathrm{FeCl}_{3}$ was obtained from PerkinElmer, Inc. through a key comparison program piloted by the National Metrology Institute of Japan (NMIJ). Two types of samples, i.e. the liquid scintillation (LS) samples and point source samples, were prepared for primary activity standardization and impurity check, respectively. The standardization was conducted using the $4 \pi \beta(\mathrm{LS})-\gamma$ anti-coincidence method and the impurities of the solution were analyzed using the gamma-spectrometry method.

\section{Sample preparation}

The LS samples were prepared in Ultima Gold $^{\mathrm{TM}}$ (UG) and Hionic Fluor (HF) cocktail solutions. Five samples for each cocktail solution were prepared by gravimetrically adding the aliquots of ${ }^{59} \mathrm{Fe}$ solution into $10 \mathrm{~mL}$ of cocktail in a $20 \mathrm{~mL}$ vial glass. Each sample series is comprised of one sample without an active solution to measure the background counting rate, which was then subtracted. The source masses on both sample series were ranged between 8 and $16 \mathrm{mg}$. In order to achieve a good homogeneous sample, all samples were shaken and kept in a dark room before they were measured. The point source samples were prepared by dropping the ${ }^{59} \mathrm{Fe}$ solution into a cellulose tape held by a ring holder.

\section{Activity standardization by $4 \pi \beta($ LS $)-\gamma$ anti- coincidence counting}

The high-efficiency $4 \pi \beta(\mathrm{LS})-\gamma \quad$ counting system developed at Korea Research Institute of Standards and Science (KRISS) in 2010 was used to measure the LS samples of ${ }^{59} \mathrm{Fe}$. The system consists of two $\mathrm{NaI}(\mathrm{Tl})$ detectors for gamma detection and a liquid scintillation detector with two PMTs for beta detection [14]. A schematic diagram of the system is shown in Fig. 2.

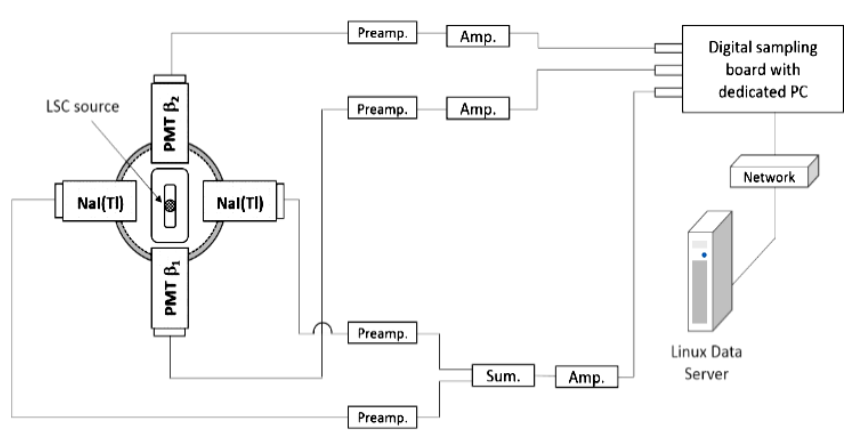

Fig. 2. Diagram of $4 \pi \beta(\mathrm{LS})-\gamma$ Coincidence Counting System.

For the current work, we applied a high voltage of $750 \mathrm{~V}$ on both gamma channels. The shaping time for both gamma and beta were set at $3 \mu \mathrm{s}$ with the threshold level set at 30 and $100 \mathrm{mV}$ for beta and gamma, respectively. A high voltage of $2600 \mathrm{~V}$ was applied for the beta anode and $700 \mathrm{~V}$ was applied for the beta dynode channel.

A digital anti-coincidence counting method was applied to determine the activity of ${ }^{59} \mathrm{Fe}$. Data acquisition was performed for 10 minutes for each sample and 10 hours for blank samples using the digital coincidence counting (DCC) technique with emulated live-time of the extending dead-time developed in 2011 [15]. This technique has been widely used with the coincidence counting method 
to overcome the difficulty in the standardization of radionuclides with a complex decay scheme. Primary activity standardization of some radionuclide, i.e. ${ }^{134} \mathrm{Cs},{ }^{137} \mathrm{Cs},{ }^{113} \mathrm{Sn},{ }^{131} \mathrm{I}$, and ${ }^{64} \mathrm{Cu}$ using digital counting technique were reported in a good agreement with other methods [16-19].

The DCC was applied in the data acquisition system to perform the offline analysis as all the information are recorded in RAW data files. RAW data files obtained from measurements were analyzed in Linux data server using programs written in FORTRAN language.

In this experiment, efficiency was varied by changing the threshold in beta channels. The rates of $\rho_{\beta}$ and $\rho_{\gamma}$ were obtained by summing up the counts from the output of beta-channel and gamma channel, respectively, and dividing the result by the live-time obtained. We determined the rates of $\rho_{\beta \gamma}$ using Eq. (1), in which the $\rho_{a}$ was determined from the anti-coincidence output. The $\rho_{\beta}, \rho_{\gamma}$, and $\rho_{a}$, were then obtained as a function of pulse height of beta discrimination level. Meanwhile, all the information from coincidence beta data file, gamma data file, and efficiency data file were extracted to construct the covariance matrix to be used as the weighting in the least-square fitting. Final data set containing the three count rates with their standard uncertainties and the matrix error for each beta discrimination level were then obtained and saved into a file. A fitting routine was used for extrapolation to the unity of beta detector and the efficiency was performed based on the information saved in the final data file. These procedures of data analysis were performed for both sample and background data. Detail procedures in data acquisition and data analysis were described in [20].

\section{RESULTS AND DISCUSSION}

Three RAW data files were obtained from the $4 \pi \beta(\mathrm{LS})-\gamma$ counting system, i.e. two data files from the beta channel and one data file from the gamma channel. Using digital coincidence counting technique, it is possible to choose any gamma window without measuring each window setting, separately. Hence, it will reduce the measuring time during the experiment.

Spectrum for each channel was obtained after the files are pre-processed with a typical spectrum as shown in Fig. 3. Based on this spectrum information, gamma window for anti-coincidence measurements was determined. Due to the correlation effect as described by Park, et al. (2000), we decided to use the gamma window at $1291.6 \mathrm{keV}$ [21]. The window was set in the program using the value of mean $\pm 2 \sigma$ obtained from the Gaussian fit line of each photopeak energy.

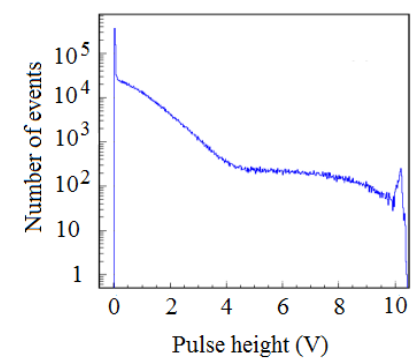

(a)

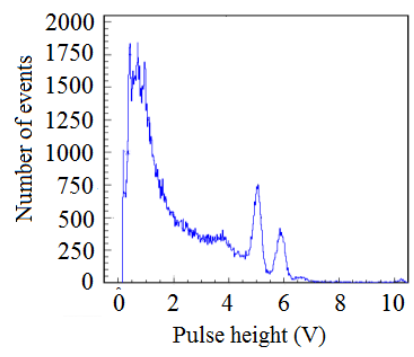

(c)

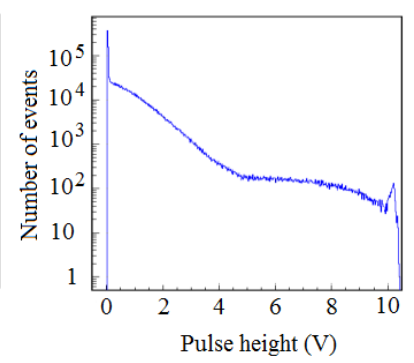

(b)

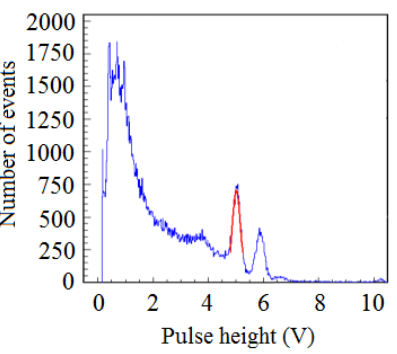

(d)
Fig. 3. Spectrum obtained from data acquisition of ${ }^{59} \mathrm{Fe}$, (a) and (b) spectrum of beta signals obtained from channel beta 1 and beta 2, respectively, (c) gamma spectrum of ${ }^{59} \mathrm{Fe}$, (d) gamma spectrum of ${ }^{59} \mathrm{Fe}$ with Gaussian fit line on $1291.6 \mathrm{keV}$ photopeak.

The activity of ${ }^{59} \mathrm{Fe}$ was determined by plotting the $\rho_{\beta} \rho_{\gamma} / \rho_{\beta \gamma}$ as a function of $\left(\rho_{\gamma} / \rho_{\beta \gamma}\right)-1$. Using the fitting routine with the least-squares method, we extrapolate the function into $100 \%$ efficiency of the beta detector to obtain the activity value. An example of the efficiency extrapolation results is shown in Fig. 4.

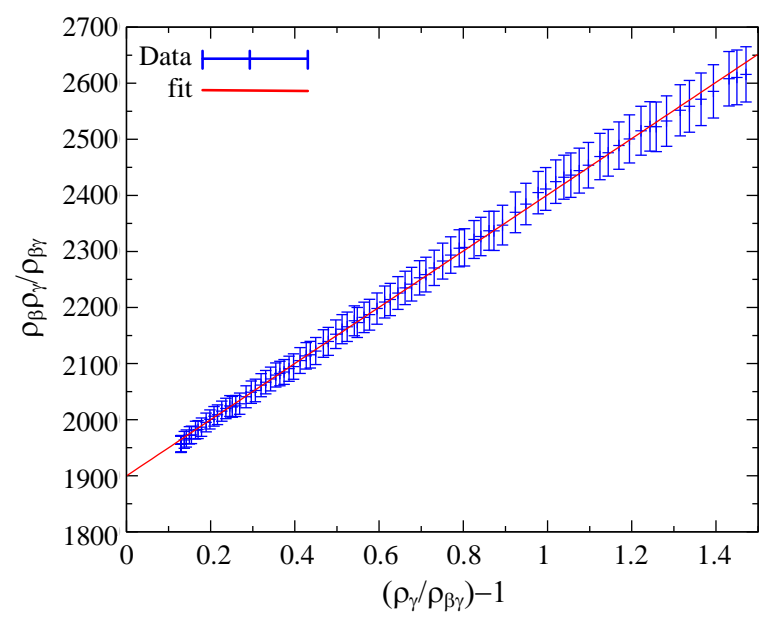

Fig. 4. Fitted efficiency extrapolation curve for ${ }^{59} \mathrm{Fe}$.

The results of UG samples provide a maximum of $93 \%$ of beta efficiency while the HF provides a maximum of $90 \%$ of beta efficiency. The difference of the efficiency range between the two cocktails was caused by the nature of the cocktails themselves, which affect the efficiency of beta detection inside the cocktail solution as explained in [22]. However, since the efficiency 
curves obtained from both sample series were extrapolated to $100 \%$ efficiency, the differences in maximum efficiency values between the two cocktails can be neglected.

The results of activity determination for both UG and HF sample series as the reference time are given in Table 1. Uncertainties were evaluated using combined standard uncertainties, which correspond to uncertainties with $k=1$.

Table 1. Results of activity standardization of ${ }^{59} \mathrm{Fe}$.

\begin{tabular}{ccc}
\hline Sample ID & Eff. Range $(\%)$ & $\begin{array}{c}\text { Specific Activity } \\
(\mathrm{kBq} / \mathrm{g})\end{array}$ \\
\hline${ }^{59} \mathrm{Fe}$ anticoin_UG & $17-93$ & $473.32 \pm 2.55$ \\
${ }^{59} \mathrm{Fe}$ _anticoin_HF & $5-90$ & $477.14 \pm 2.42$ \\
\hline
\end{tabular}

In Table 1, we can see that there is a $0.80 \%$ difference between UG and HF samples, which might come from the instability of UG cocktails as explained by Fitzgerald et al. (2014) [23]. However, this level of difference is still acceptable and in a good agreement with the results when we expand the uncertainty coverage factor to $k=2$. The uncertainty budgets for activity determination of ${ }^{59} \mathrm{Fe}$ is given in Table 2 .

Table 2. Relative standard uncertainty budget for the activity standardization of ${ }^{59} \mathrm{Fe}$.

\begin{tabular}{lccc}
\hline \multirow{2}{*}{\multicolumn{1}{c}{ Parameter }} & Type & \multicolumn{2}{c}{$\begin{array}{c}\text { Relative standard } \\
\text { uncertainty }(\%)\end{array}$} \\
\cline { 3 - 4 } & & UG & HF \\
\hline SD of counting & $\mathrm{A}$ & 0.18 & 0.15 \\
Weighing & $\mathrm{B}$ & 0.14 & 0.14 \\
Half-life of ${ }^{59} \mathrm{Fe}$ & $\mathrm{B}$ & 0.02 & 0.02 \\
Impurities of ${ }^{59} \mathrm{Fe}$ & $\mathrm{B}$ & 0.02 & 0.02 \\
Eff. Extrapolation & $\mathrm{B}$ & 0.35 & 0.35 \\
$\beta-\beta$ resolving time & $\mathrm{B}$ & 0.30 & 0.30 \\
\hline Combined standard & & 0.51 & 0.51 \\
uncertainty & & 1.02 & 1.02 \\
Expanded uncertainty & & &
\end{tabular}

The highest contributor to uncertainty in this experiment was from the efficiency extrapolation with a value of $0.35 \%$. This is because the systematic uncertainty coming from the data acquisition system creates scatter in the data sets. In addition, it is also affected by the estimated uncertainty due to the variation of the source masses, which enter the efficiency extrapolation as explained in [24]. Impurities of the master solution of ${ }^{59} \mathrm{Fe}$ were also taken into account in the uncertainty evaluation. The value was obtained from gamma-ray spectrometry analysis on point source samples, which show an impurity of ${ }^{60} \mathrm{Co}$ in the solution with an activity of $(91 \pm 7) \mathrm{Bq} / \mathrm{g}$. The activity ratio of ${ }^{60} \mathrm{Co}$ to ${ }^{59} \mathrm{Fe}$ at the reference date is approximately $0.02 \%$.
The final activity results were compared and found to be in a good agreement with the result from $4 \pi \beta(\mathrm{LS})-\gamma$ and $4 \pi \beta(\mathrm{PC})-\gamma$ coincidence counting method, which respectively reported the final activity of ${ }^{59} \mathrm{Fe}$ as $(472.06 \pm 3.00) \mathrm{kBq} / \mathrm{g}$ and $(471.80 \pm 3.45) \mathrm{kBq} / \mathrm{g}$, with quoted uncertainty evaluated at $k=1$.

\section{CONCLUSION}

The primary activity of ${ }^{59} \mathrm{Fe}$ has been successfully determined using the digital anticoincidence counting method with emulated livetime of the extending dead-time applied to the $4 \pi \beta(\mathrm{LS})-\gamma$ counting system. The final activity found by this method is in good agreement with an uncertainty level measured by the coincidence counting method. This result shows that the digital anti-coincidence counting method can be used as an alternative method for primary activity determination of ${ }^{59} \mathrm{Fe}$.

\section{ACKNOWLEDGMENT}

The authors are indebted to the Korea Research Institute of Standards and Science (KRISS) for supporting this research activity.

\section{REFERENCES}

1. M. Sahagia, Role of the Radionuclide Metrology in Nuclear Medicine, in: A. Gholamrezanezhad (Ed.), 12 Chapters Nucl. Med., Intechopen, Rijeka, Croatia (2011) 111.

2. M. Bé, V. Chisté, C. Dulieu et al., Table of Radionuclides, BIPM, Sèvres (2004) 99.

3. A. Agusbudiman, K.B. Lee, J.M. Lee et al., Standardization of ${ }^{59} \mathrm{Fe}$ by $4 \pi \beta(L S)-\gamma$ Coincidence Counting Method, Proceedings of 2nd International Conference on Sources, Effects, and Risk Ionizing Radiation (2016) 59.

4. M.F. Koskinas, G. Polillo, F. Brancaccio et al., Appl. Radiat. Isot. 109 (2016) 386.

5. M. Zhang, S. Yao, J. Liang et al., Appl. Radiat. Isot. 109 (2016) 341.

6. M.W. van Rooy, M.J. van Staden, J. Lubbe et al., Appl. Radiat. Isot. 109 (2016) 276.

7. T. Yamada, Y. Kawada and Y. Sato, Appl. Radiat. Isot. 109 (2016) 414.

8. K. Kossert and O.J. Nähle, Appl. Radiat. Isot. 93 (2014) 33.

9. E. García-Toraño, T. Altzitzoglou, P. Auerbach et al., Appl. Radiat. Isot. 140 
(2018) 157.

10. C.J. da Silva, A. Iwahara, R. Poledna et al., Appl. Radiat. Isot. 66 (2008) 886.

11. C.J. da Silva, P.A.L. da Cruz, A. Iwahara et al., Appl. Radiat. Isot. 134 (2018) 307.

12. C.J. da Silva, E.M. de Oliveira, A. Iwahara et al., Appl. Radiat. Isot. 87 (2014) 188.

13. C.J. da Silva, P.A.L. da Cruz, A. Iwahara et al., Appl. Radiat. Isot. 134 (2018) 316.

14. K.B. Lee, J.M. Lee, T.S. Park et al., IEEE Trans. Nucl. Sci. Proc. 57 (2010) 2613.

15. K.B. Lee, J.M. Lee, T.S. Park et al., IEEE Nucl. Sci. Symp. Conf. Rec. (2011) 873.

16. D.B. Kulkarni, R. Anuradha, L. Joseph et al., Appl. Radiat. Isot. 108 (2016) 24.

17. J. Sochorová and P. Auerbach, Appl. Radiat. Isot. 87 (2014) 207.
18. D.E. Bergeron, J.T. Cessna, R. Fitzgerald et al., Appl. Radiat. Isot. 139 (2018) 266.

19. M. Roteta, V. Peyres, E. García-Toraño, Appl. Radiat. Isot. 87 (2014) 162.

20. K.B. Lee, J.M. Lee, T.S. Park et al., Nucl. Instruments Methods Phys. Res. Sect. A Accel. Spectrometers, Detect. Assoc. Equip. 626 (2011) 72.

21. T.S. Park, H.Y. Hwang and J.M. Lee, Appl. Radiat. Isot. 52 (2000) 435.

22. P. Cassette, Appl. Radiat. Isot. 87 (2014) 32.

23. R. Fitzgerald, B.E. Zimmerman, D.E. Bergeron et al., Appl. Radiat. Isot. 85 (2014) 77.

24. C.J. Bailat, J. Keightley, Y. Nedjadi et al., Metrologia 5106018 (2014). 